\title{
Surgical audit of a single-surgeon experience with laparoscopic cholecystectomy in northern Saskatchewan, Canada
}

\section{Bernado Verdolin \\ Yagan Pillay ${ }^{2}$}

'Hospital dos Servidores do Estado do Rio de Janeiro, Rua Sacadura Cabral, Brazil; ${ }^{2}$ University of Saskatchewan College of Medicine, General Surgery, Saskatchewan, Canada
Correspondence: Yagan Pillay University of Saskatchewan College of Medicine, General Surgery, 3310 6th Avenue West Prince Albert, S6V 8C3 Saskatchewan, Canada Email yagan2pillay@yahoo.ca
This article was published in the following Dove Press journal:

Clinical Audit

Aims: This study reviewed laparoscopic cholecystectomy in the clinical practice of a single general surgeon in northern Saskatchewan, Canada.

Methods: Data were accumulated from the electronic medical records (EMRs) software and health records (HRs) department of the Victoria Hospital. This audit searched for all laparoscopic cholecystectomies between the years 2009 and 2016 inclusive.

Results: Two hundred and ninety laparoscopic cholecystectomies were performed over 7 years. They were analyzed for various aspects such as age, with the majority of patients between 41 and 65 years, and gender, with the female gender in $71.8 \%$ of cases. Our complication rates in both elective and emergency procedures, along with conversion $(1.37 \%)$ and reoperation $(1.03 \%)$ rates, were examined. The lengths of stay in elective (1.02 days) and emergency (2.13 days) surgeries were comparable to the reported literature. We examined associated procedures performed with the laparoscopic cholecystectomy, procedure duration, converted surgeries, and reoperated patients, as well as the referral rate of patients to a quaternary care hospital for super specialist evaluation and management not available locally. Biliary leakage was diagnosed in four cases postoperatively $(1.37 \%)$, showing similar trends to published literature rates varying from $0.56 \%$ to $3.5 \%$.

Conclusion: In Prince Albert Parkland health region, even with limited resources in a small city such as Prince Albert in northern Saskatchewan and great distances between the two health care centers, it is possible to provide a high standard of surgical care for the population with results comparable to published international standards in minimally invasive gallbladder surgery.

Keywords: surgical audit, laparoscopic cholecystectomy, rural surgery, audit database

\section{Introduction}

Cholelithiasis and its attendant clinical sequelae remain a common pathology in North America affecting $10 \%-15 \%$ of the adult population. Approximately $20-25$ million Americans have cholelithiasis. ${ }^{1}$ The prevalence among First Nations women in Canada has reached $62 \%$, one of the highest reported rates worldwide. ${ }^{1}$

There are a considerable number of complications due to cholelithiasis such as severe acute pancreatitis, cholangitis, and emphysematous cholecystitis and may require emergency surgical treatment. Other complications may cause unusual clinical scenarios such as a bowel obstruction due to chronic cholecystitis and gallbladder fistulae ${ }^{2}$ or jaundice due to a common bile duct (CBD) obstruction.

Surgery remains the treatment of choice for acute disease that affects the gallbladder and the CBD. ${ }^{3-6} \mathrm{~A}$ few complications can be managed non-operatively with interven- 
tions such as percutaneous cholecystostomy, endoscopic retrograde cholangiopancreatography (ERCP), and placement of a stent inside the CBD for calculi $<2 \mathrm{~cm}$ or even pneumatic lithotripsy for impacted calculi $>2 \mathrm{~cm} .^{3}$

Laparoscopic surgery has significantly changed the perioperative landscape of gallbladder disease and has become the recognized standard worldwide since its introduction in $1985 .^{7}$

Recently, a significant number of studies in the published literature have shown a trend toward conservative treatment as an alternative to surgery. ${ }^{4}$ This has been disputed in a recent study by de $\mathrm{Mestral}^{4}$ and coworkers comparing the cost of three different treatment options. He concluded that the least expensive management, with better clinical outcomes, for uncomplicated acute cholecystitis is laparoscopic surgery. ${ }^{4,5}$ This is a reasonable observation in this era of financial jurisprudence.

\section{Methods}

In this retrospective audit, we examined the experience of a single general surgeon in the Prince Albert Parkland Health Region (PAPHR) performing laparoscopic surgery for gallbladder disease. This study included all laparoscopic cholecystectomies between the years 2009 and 2016 inclusive performed at the Victoria Hospital in Prince Albert, Saskatchewan. The Victoria Hospital serves a mixed population of urban and rural communities in northern Saskatchewan, Canada, and provides a quality service with a specialized team of laparoscopic surgeons.

The research was registered with and approved by the PAPHR Ethics Committee. The ethics committee did not require patient consent as this was a chart review without identifying the individual patients. All patient information data were securely stored in an Excel $^{\circledR}$ spreadsheet with password protection.

The audit gathered data from the electronic medical records (EMR) software that has been introduced at the hospital since 2010. The data were acquired retrospectively for the same period. Direct chart review in the health records (HRs) department of the Victoria Hospital was another tool used to obtain detailed and accurate information about patient's admission, surgical procedures, and outcomes throughout the 8 years studied. In addition, charts were examined electronically in the surgeon's EMR at his office.

All symptomatic patients with gallstone disease who had laparoscopic surgery were considered for this study, including those who needed conversion to an open procedure. Exclusion criteria were primarily any open cholecystectomy procedure performed as a first choice without any attempt at a laparoscopic approach. Variables included demographic data such as gender and age; elective and emergency procedures; complications, conversion rates, the length of admission, and procedures performed concomitantly with the cholecystectomy; operative time; and referral rate of patients to a quaternary care hospital. The surgery duration was measured in minutes, and length of hospital admission was recorded in days.

Day care surgery, same day admission, and discharge were considered as 0 days. Referrals to a quaternary hospital were due to clinical or surgical complications needing a specialist consult such as an elective or urgent ERCP or to an interventional radiologist (IR) for a percutaneous cholecystostomy. None of these interventions are available at our hospital.

\section{Results}

The patient's age ranged between 11 and 82 years (Figure 1).

Two hundred and eight procedures were performed on female patients $(71.8 \%)$, while $82(28.2 \%)$ male patients were subjected to laparoscopic cholecystectomy (Figure 2).

There were 290 cases considered for this study, of which 206 were elective and 84 were emergency procedures. No patient had an intraoperative CBD exploration during the primary surgery. ERCP was performed at the quaternary referral hospital perioperatively to manage 11 cases of symptomatic

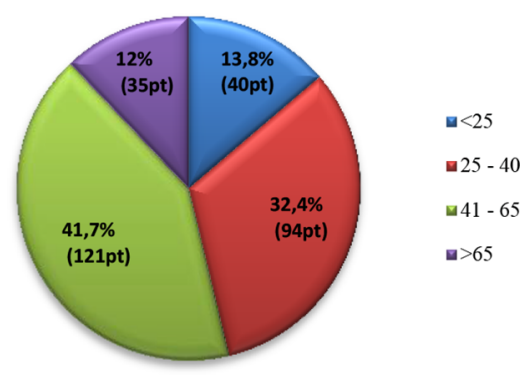

Figure I Age distribution of patients.

Abbreviation: pt, patient.

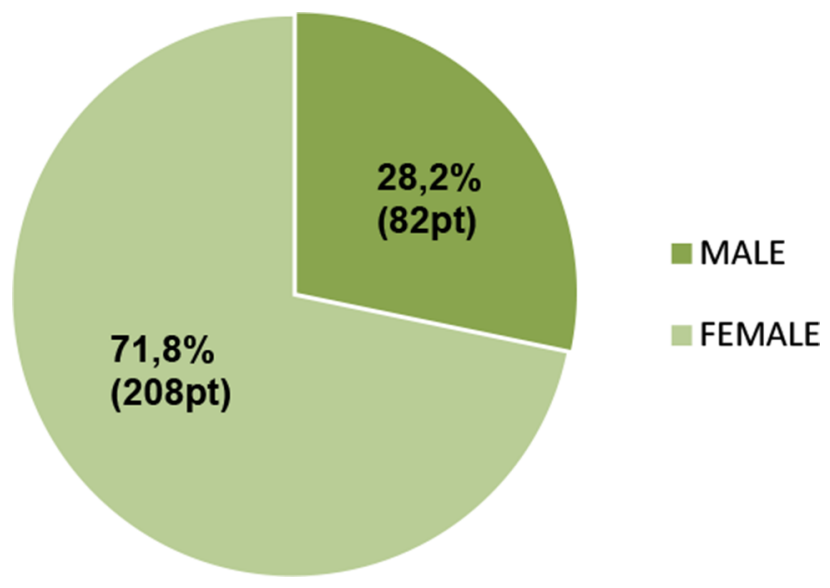

Figure 2 Gender distribution of the patient cohort. Abbreviation: pt, patient. 
choledocholithiasis and three cases of surgical complications involving the biliary tree (Figure 3). Eight were done preoperatively and six postoperatively.

Complications were diagnosed in 23 of the 290 patients $(8.27 \%), 8$ of whom were males and 15 were females (Figure 4 ). There were 11 cases of surgical complications, seven isolated wound complications and five cases of clinical complications.

The Clavien-Dindo classification ${ }^{8}$ was used to classify complications based on the surgical technique or clinical evolution (Table 1).

\section{Grade I}

We had six wound infections and two wound hematomas that healed with conservative treatment. Two of these wound

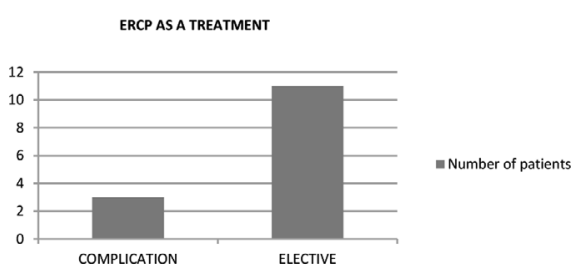

Figure 3 Number of patients treated with ERCP for choledocholithiasis and postoperative complications.

Abbreviation: ERCP, endoscopic retrograde cholangiopancreatography.

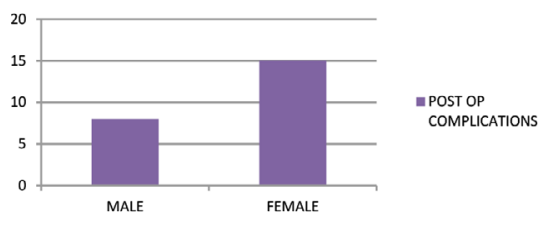

Figure 4 Complications according to the gender. infections were emergency cases, and only one of them had an associated complication (small bowel perforation). Two patients claiming abdominal discomfort were treated with conventional analgesia. A colonic perforation was repaired intraoperatively and did not change the patient's outcome during this admission. A superior epigastric vein laceration was treated intraoperatively with bipolar coagulation. There was one case of readmission on the same day of discharge due to an intraoperative cystic artery bleeding that presented with hypotension, tachycardia, and nausea postoperatively. This was managed conservatively with no surgical intervention.

\section{Grade II}

Five of our clinical complications were classified as Grade II. A urinary tract infection was treated with antibiotics, and another patient with postoperative urinary retention required a Foley's catheterization. One patient developed a myocardial infarction and was referred to a quaternary hospital. Another patient developed a small localized biloma that was managed conservatively with antibiotics. The last patient in this group developed bleeding from a middle hepatic vein injury that required conversion to an open procedure with vein ligation, blood transfusion, and subsequent transfer to a hepatobiliary surgeon at the referral quaternary center. Both the cardiac patient and the patient with the middle hepatic vein injury made uneventful recoveries.

\section{Grade III}

Four of five biliary leaks were classified as Grade III. Two of them (CBD injury and bile peritonitis) needed a laparotomy (Grade IIIb) and two others required ERCP and percutaneous

Table I Clavien-Dindo ${ }^{\odot}$ classification of surgical complications

\begin{tabular}{|c|c|}
\hline Grade & Definition \\
\hline Grade I & $\begin{array}{l}\text { Any deviation from the normal postoperative course without the need for pharmacological treatment or surgical, endoscopic, } \\
\text { and radiological interventions } \\
\text { Allowed therapeutic regimens are drugs such as antiemetics, analgesics, diuretics, electrolytes, and physiotherapy. This grade } \\
\text { also includes wound infections opened at the bedside }\end{array}$ \\
\hline Grade II & $\begin{array}{l}\text { Requiring pharmacological treatment with drugs other than such allowed for Grade I complications } \\
\text { Blood transfusions and total parenteral nutrition are also included }\end{array}$ \\
\hline Grade III & Requiring surgical, endoscopic, or radiological intervention \\
\hline Grade Illa & Intervention not under general anesthesia \\
\hline Grade IIIb & Intervention under general anesthesia \\
\hline Grade IV & Life-threatening complications (including CNS complications) ${ }^{\mathrm{a}}$ requiring IC/IUC management \\
\hline Grade IVa & Single-organ dysfunction (including dialysis) \\
\hline Grade IVb & Multiorgan dysfunction \\
\hline Grade V & Death of a patient \\
\hline Suffix “d” & $\begin{array}{l}\text { If the patient suffer from a complication at the time of discharge (see examples in Table } 2 \text { ), the suffix "d" (for "disability") is } \\
\text { added to the respective grade of complication. This label indicates the need for a follow-up to fully evaluate the complication }\end{array}$ \\
\hline
\end{tabular}

Note: aBrain hemorrhage, ischemic stroke, subarachnoid bleeding, but excluding transient ischemic attacks.

Abbreviations: CNS, central nervous system; IC, intermediate care; ICU, intensive care unit. 
drainage (Grade IIIa). The fifth case, a small bowel perforation, required a reoperation 2 days postlaparoscopic cholecystectomy.

There were no Grade IV and Grade V complications.

The summary of our complications (Figure 5) and its distribution based on the Clavien-Dindo's classification are shown below (Table 2).

Emergencies were responsible for 8 of the 23 complications $(35 \%)$, leading to a relaparotomy in three patients (Figure 6). One was a small bowel injury diagnosed on the third day after discharge and managed at our hospital and two referred cases of biliary leakage and CBD stricture.

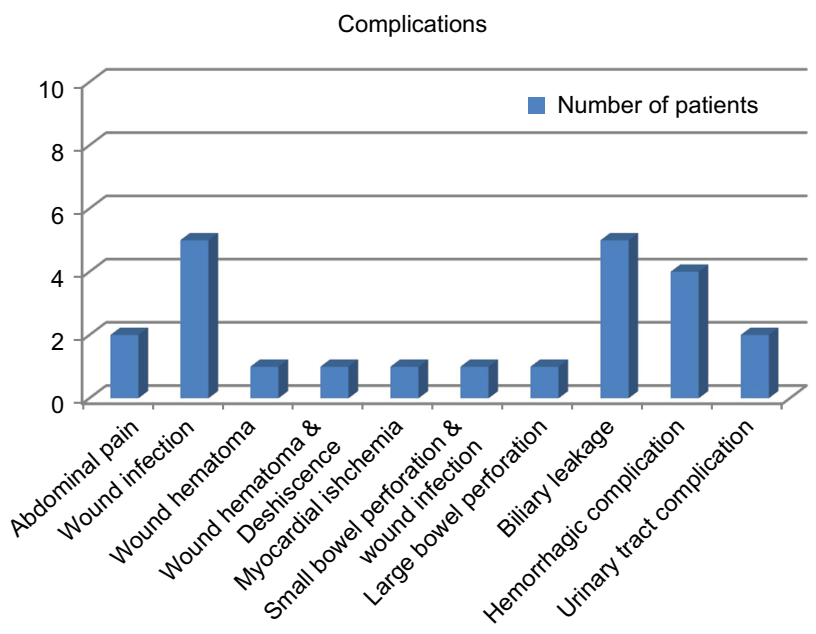

Figure 5 Number of complications.

Table 2 Summary of complications based on the Clavien-Dindo ${ }^{\odot}$ classification

\begin{tabular}{|l|l|l|}
\hline Clavien-Dindo & Total & Complication \\
\hline Grade I & I3 & $\begin{array}{l}\text { 6 wound infections } \\
\text { 2 wound hematomas } \\
\text { I colonic perforation } \\
\text { I superior epigastric vein laceration } \\
\text { I postoperative hypotension } \\
\text { 2 abdominal pain }\end{array}$ \\
\hline Grade II & 5 & $\begin{array}{l}\text { I urinary tract infection } \\
\text { I urinary retension } \\
\text { I myocardial infarction } \\
\text { I biloma } \\
\text { I hepatic vein injury }\end{array}$ \\
\hline Grade IIla & 3 & $\begin{array}{l}\text { 2 biliary leakage } \\
\text { I small bowel perforation }\end{array}$ \\
\hline Grade IIIb & 2 & $\begin{array}{l}\text { I CBD injury } \\
\text { I biliary peritonitis }\end{array}$ \\
\hline Grade IV and V & 0 & \\
\hline
\end{tabular}

Abbreviation: $\mathrm{CBD}$, common bile duct.
Intraoperative complications were managed laparoscopically in cases of uncontrolled diffuse bleeding from the hepatic parenchyma in a cirrhotic liver (one patient) and uncontrolled bleeding from the cystic artery (one patient).

Conversion to an open procedure was performed in four patients: two for intraoperative complications such as a large bowel perforation and diffuse bleeding from a middle hepatic vein injury and two intraoperative decisions due to chronic inflammation and difficulty with dissection (a Mirizzi syndrome and chronic cholecystitis with a shrunken gallbladder). The referral rate for complicated cases was $21.7 \%$ (5 of 23 cases).

The average operating time was 93 minutes (range, 35-261 minutes). The average time for emergency procedures and converted procedures increased in comparison to that for elective surgery (99.6 and 180.5 minutes, respectively; Figure 7). This is similar to the referenced literature where it was observed that in procedures on high-risk patients (men, the elderly, emergency cases, and multiple comorbidities), the operative time increased from less than 70 minutes for elective cases to between 75 and 114 minutes in non-elective cases. ${ }^{10}$

Major hemorrhage in four cases $(1.37 \%)$ was directly related to an increased surgical time, varying from 66 to 194

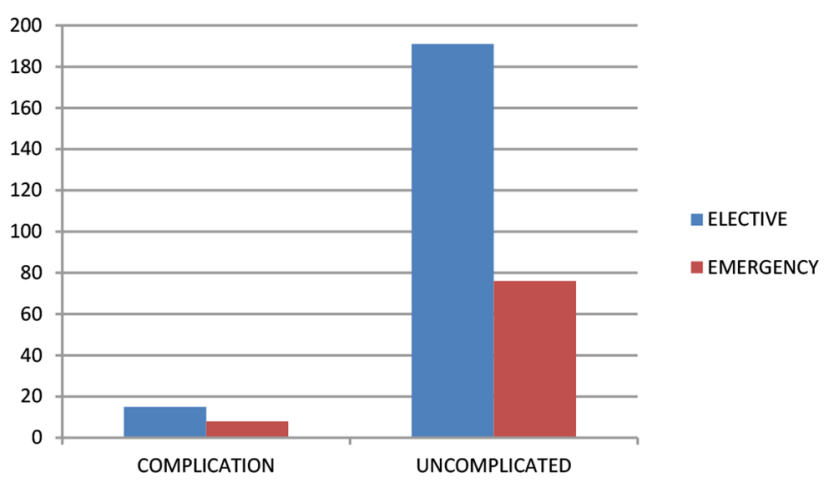

Figure 6 Number of patients with complications and without complications submitted for elective and emergency procedures.

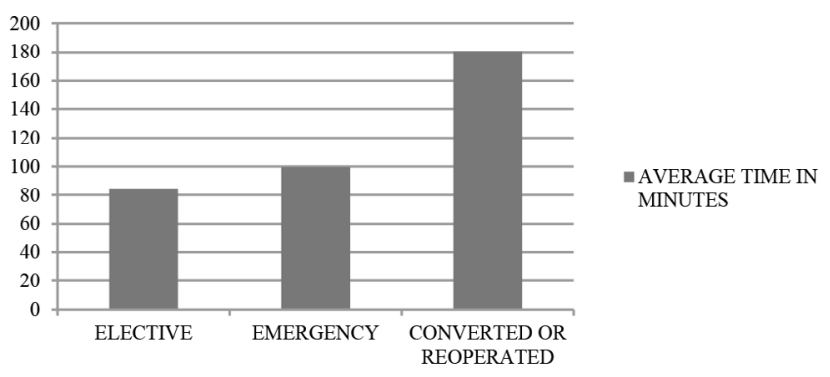

Figure 7 Average time in minutes for elective, emergency, and converted or reoperated cholecystectomy. 
minutes (mean of 134.5 minutes). Three of them were managed with the use of clips, electrocautery, and Surgicel $^{\odot}$, and only one (a middle hepatic vein injury) required conversion to an open surgery for a hemostatic suture. In comparison with the study by Duca et al, ${ }^{9}$ there is a reduced incidence of hemorrhagic complications ( $1.37 \%$ vs $2.3 \%$ ). Biliary leakage was diagnosed in four cases postoperatively (three bilomas and one bile peritonitis; $1.37 \%$ ), and CBD stricture was diagnosed in one patient who presented with clinical jaundice $(0.34 \%)$. Their management did not change the mean surgical time, which remained similar in all emergency surgeries (99.2 minutes). The average biliary leak rate in referenced literature varied in different studies $(0.56 \%-3.5 \%),{ }^{9,10}$ but the rate of biliary tree injuries was superior than our study $(0.1 \%$ vs $0.34 \%){ }^{8}$

The average length of stay was 1.02 days. The lengths of stay for converted and reoperative surgeries were 4.25 and 19.6 days, respectively. In complicated cases, the average duration was 5.2 days (Figures 8 and 9).

\section{Discussion}

Emergencies represented $28.9 \%$ of overall patients treated for gallbladder disease.

Eight were evaluated with six clinical and two surgical complications. No emergency patient needed conversion to

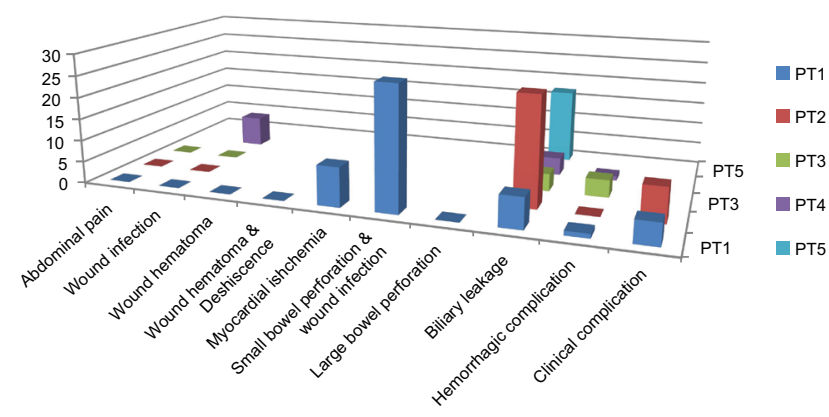

Figure 8 Length of stay in days for patients with complications.

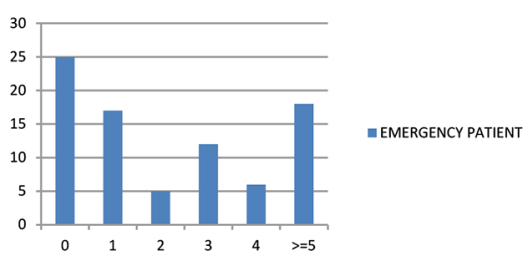

Figure 9 Number of emergency patient per number of days in hospital. open surgery, a considerable difference from the reported literature, which describes emergency procedures as a potential risk factor for conversion to open surgery when compared to elective surgery. ${ }^{9-11}$

It was observed that the average length of stay for patients with emergency procedures increased from 1.02 days for elective cases to 2.13 days in emergency surgery. The surgical time of the procedure also increased in acute surgery by an additional 8 minutes when compared to the operative time for an elective procedure.

Four of the 206 elective procedures needed conversion to an open cholecystectomy. Two of them were due to a frozen Calot's triangle and difficult laparoscopic visualization (Mirizzi syndrome and chronic cholecystitis), and two were due to intraoperative complications (colonic perforation and middle hepatic vein injury). Our conversion rate to open surgery in elective laparoscopic cholecystectomy was $1.37 \%$, which is below the average of published studies with reported conversion rates of $2 \%, 5.9 \%$, and $15 \% .^{9-11}$ In addition, the rate of reoperation was $1.03 \%$ in all patients. The overall complication rate compared with the published experience of other centers was similar at $7.9 \%$. Our mortality rate was zero.

ERCP was performed in 14 cases including 11 for choledocholithiasis and three for biliary leakage. All cases of gallstone pancreatitis or post-ERCP pancreatitis were treated at the Victoria Hospital. IR was required in the management of biloma showing excellent results and reducing the number of relaparotomies (Figure 10). We did not have access to pneumatic lithotripsy as an option for choledocholithiasis; however, no surgical exploration of the $\mathrm{CBD}$ was required due to ERCP failure. The endoscopic management of choledocholithiasis has shown commendable results in our community.

A single iatrogenic stricture lesion was diagnosed in the surgical cohort and classified as CBD injury Strasberg E4.

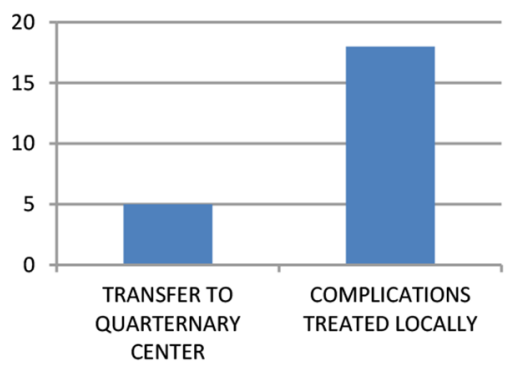

NUMBER OF PATIENTS WITH COMPLICATIONS

Figure 10 Patients with complications that needed transfer to a quaternary center. 
All other cases of biliary leakage that were diagnosed and treated were classified as Strasberg type A. ${ }^{12}$ The original analysis of biliary reconstruction was based on the Bismuth classification and subsequently modified by Strasberg.

None of the 290 patients developed a port site hernia, with reported incidences in the literature of $0.125 \% .{ }^{9}$ This may have been due to the intraoperative decision to close the fascial defect of the infraumbilical port as most of the reported port site hernias occur through this port site. One disadvantage of this audit is that we did not have access to the long-term follow-up of our patients due to lack of integration between the family physician notes in remote areas of the province and the EMR database in Prince Albert. This exposure to the patient's medical records may have facilitated better long-term follow-up in determining the long-term incidence of port site hernias.

A disturbing trend in our study is the number of cholecystectomies in patients younger than 40 years. They made up $46.2 \%$ of the total surgeries, and if this trend continues to increase, then there is certainly a cause for concern. This incidence is higher than the numbers found in a British study ${ }^{13}$ that showed $21 \%$ of symptomatic gallbladder stones in patients younger than 40 years. The pathology in these cases was due to cholesterol stones unlike in developing nations where pigment stones make up a large number in young patients. This is most certainly attributable to poor lifestyle choices in terms of nutrition and fueled no doubt by the increasing number of childhood obesity rates in the developed world. ${ }^{1}$

We would also like to put forward this audit as the start of a Canada-wide database for practicing surgeons, so that we may have access to surgical standards across the country. This is to ensure that we maintain our clinical standards especially in rural practice where it remains far more difficult to update our surgical skill set on a regular basis.

We envisage a website for provincial and municipal surgical audits that will serve as a database for maintaining surgical standards. In this era of professional accountability and public transparency, this would seem a wise investment in our surgical craft.

\section{Conclusion}

Despite working in a rural center in northern Saskatchewan with limited resources and a large geographic area, a multidisciplinary team can be accessed to offer a universally acceptable treatment for all patients while maintaining internationally recognized surgical standards as per clinical guidelines and the best available evidence to date.

\section{Disclosure}

The authors report no conflicts of interest in this work.

\section{References}

1. Stinton LM, Shaffer EA. Epidemiology of gallbladder disease: cholelithiasis and cancer. Gut Liver. 2012;6(2):172-187.

2. Pillay Y, Gallstones W. Gallbladder Fistulae. Austin Surg Case Rep. 2016;1(1):1003

3. Farooq Qadri SJ, Khan M, Khan N, Syed Javid Farooq Qadri, Khan N. Use of pneumatic lithotripsy for managing difficult CBD calculi. Int $J$ Surg. 2011;9(1):59-62.

4. de Mestral C, Hoch JS, Laupacis A, et al. Early Cholecystectomy for Acute Cholecystitis Offers the Best Outcomes at the Least Cost: A Model-Based Cost-Utility Analysis. J Am Coll Surg. 2016;222(2):185-194.

5. Gutt CN, Encke J, Köninger J, et al. Acute cholecystitis: early versus delayed cholecystectomy, a multicenter randomized trial (ACDC study, NCT00447304). Ann Surg. 2013;258(3):385-393.

6. Bansal VK, Misra MC, Rajan K, et al. Single-stage laparoscopic common bile duct exploration and cholecystectomy versus two-stage endoscopic stone extraction followed by laparoscopic cholecystectomy for patients with concomitant gallbladder stones and common bile duct stones: a randomized controlled trial. Surg Endosc. 2014;28(3):875-885.

7. The Southern surgeon's Club. A Prospective Analysis Of 1518 Laparoscopic Cholecystectomies. N Engl J Med. 1991;324(16): 1073-1078.

8. Daniel Dindo MD, Nicolas Demartines MD, Clavien P-A. Classification of Surgical Complications - A New Proposal with Evaluation in a Cohort of 6336 Patients and Results of a Survey. Annals of Surgery. 2004;240(Number 2).

9. Duca S, Bãlã O, Al-Hajjar N, et al. Laparoscopic cholecystectomy: incidents and complications. A retrospective analysis of 9542 consecutive laparoscopic operations. HPB. 2003;5(3):152-158.

10. Pavlidis TE, Marakis GN, Ballas K, et al. Risk factors influencing conversion of laparoscopic to open cholecystectomy. $J$ Laparoendosc Adv Surg Tech A. 2007;17(4):414-418.

11. Karna NA, Kologlu M, Doganay M, et al. A Risk Score For Convertion From Laparoscopic To Open Cholecystectomy. Am J Surg 1001 Jun; 181(6):520-525.

12. Strasberg SM, Hertl M, Soper NJ. An analysis of the problem of biliary injury during laparoscopic cholecystectomy. J Am Coll Surg. 1995;180(1):101-125.

13. Kanakala V, Borowski DW, Pellen MG, et al. Risk factors in laparoscopic cholecystectomy: a multivariate analysis. Int J Surg. 2011;9(4):318-323. 
Clinical Audit is an international, peer-reviewed, open access journal focusing on the processes and outcomes of clinical audit in any area of healthcare. All aspects of patient care are addressed within the journal and practitioners from all disciplines are invited to submit their work. Areas covered include: Publication of audits; How an audit has changed practice;

Submit your manuscript here: https://www.dovepress.com/clinical-audit-journal
Dovepress

Practical tips on how to do audits and to avoid pitfalls; How audits have changed patient care; Calls and justifications for new audits. The manuscript management system is completely online and includes a very quick and fair peer-review system, which is all easy to use. Visit http://www.dovepress. com/testimonials.php to read real quotes from published authors. 